\title{
Dans $x$ temps est-il déictique ? Ou dans deux jours est-il à deux jours plus tard ce que demain est à le lendemain?
}

\author{
Anne Le Draoulec et Marcel Vuillaume
}

\section{$0 \quad$ Introduction}

Dans « La nature des pronoms», Benveniste (1966: 253-254) évoque les « corrélations» qui, selon lui, existent entre les "indicateurs" et les termes qui leur correspondent, mais qui "se réfèrent, non plus à l'instance de discours, mais aux objets "réels", aux termes et lieux "historiques" ». Parmi ces corrélations, il mentionne notamment: aujourd'hui : le jour même, hier: la veille, demain : le lendemain, la semaine prochaine : la semaine suivante, il y a trois jours : trois jours avant.

On reconnaît ici aisément deux séries ${ }^{1}$, celle des indexicaux - aujourd'hui, hier, demain, la semaine prochaine, il y a trois jours - et celle des termes qui, selon la curieuse formule de Benveniste, réfèrent aux « objets réels »-le jour même, la veille, le lendemain, la semaine suivante, trois jours avant. Ces termes sont communément considérés comme anaphoriques, parce que leur emploi requiert un contexte dont on puisse extraire par anaphore un repère permettant l'identification de leur référent. Cette propriété se manifeste notamment par le fait qu'ils ne peuvent apparaître à l'initiale d'un discours.

Quant aux corrélations dont parle Benveniste, elles rappellent évidemment les «proportions » connues des grammairiens de l'Antiquité ${ }^{2}$ : la veille est à hier ce que le lendemain est à demain ou ce que la semaine suivante est à la semaine prochaine, etc. Or, si l'on essaie de prolonger cette énumération, on constate que Benveniste ne mentionne pas la relation qui semble associer dans deux heures (qu'on représentera désormais par le sténogramme plus général dans $x$ temps) à deux heures plus tard ( $x$ temps plus tard), et on peut se demander si cette omission est fortuite ou si elle est dûment motivée.

Disons tout de suite que $x$ temps plus tard est un anaphorique et a donc sa place dans la série citée supra. En revanche, le statut de dans $x$ temps est beaucoup moins clair, mais n'a pourtant guère retenu l'attention des linguistes. Certains le considèrent sans discussion comme un indexical (Moeschler 1993 : 44), alors que d'autres sont plus nuancés. Henry (1968 : 116-117) écrit :

«Dans temporel [nous place] dans une prospective qui s'insère, soit dans le futur, soit dans le passé [...] prospective vue du nynégocentrique véritable ou du nynégocentrique transposé dans le passé par l'indirect libre. »

Pinchon (1974: 54), de son côté, considère que dans $x$ temps ne peut se rapporter qu'au moment de la parole, mais précise néanmoins (en se référant à Henry) que, dans le discours indirect libre, il peut être relatif à un repère passé. Berthonneau (1989) suggère elle aussi cette double possibilité pour dans $x$ temps de renvoyer, soit au "repère-origine", soit à la «translation » dans le passé de ce repère (p. 232) - tout en continuant dans tous les cas de parler de «dans-repère déictique $»^{3}$. Enfin, Schlenker (2005: 47-48) note que dans $x$ temps peut être interprété par rapport au contexte d'un acte de parole rapporté et que ce n'est donc pas un "indexical kaplanien » comme demain, mais il le considère quand même comme un indexical parce que « sa référence dépend du contexte d'énonciation ».

Chez Mc Taggart, le indexicaux correspondent à la série $\mathrm{A}$, les anaphoriques à la série $\mathrm{B}(C f$. BourgeoisGironde, 2000).

Cf. Douay \& Pinto (1991).

Par opposition avec le «dans-espace de temps » de dans la journée. 
Ces observations pointent clairement le principal obstacle qui interdit de ranger dans $x$ temps dans la même catégorie que demain, à savoir la possibilité de l'employer dans le discours indirect et de l'interpréter par rapport à un repère distinct du moment de son énonciation effective. Mais elles traitent le problème de façon beaucoup trop succincte pour qu'on puisse le considérer comme réglé ${ }^{4}$. Notre propos sera donc, sinon de trancher définitivement la question du statut de dans $x$ temps, du moins de mettre à la disposition du lecteur toutes les pièces d'un dossier pour l'instant incomplet.

Nous nous orienterons à partir de la proportion présumée ${ }^{5}$ :

$\frac{\text { demain }}{\text { le lendemain }}=\frac{\text { dans } x \text { temps }}{x \text { temps plus tard }}$

et nous comparerons dans $x$ temps aux deux termes avec lesquels il est en relation : son homologue demain et son partenaire $x$ temps plus tard. Dans une première partie, nous examinerons les arguments qui s'opposent à l'idée que dans $x$ temps puisse être un indexical. Puis dans une deuxième partie, nous procèderons à une comparaison systématique des emplois de dans $x$ temps et de demain, en distinguant au préalable les différents types de contexte dans lesquels ils apparaissent, car l'importance de ce paramètre reste largement méconnue et, comme nous le verrons, cette méconnaissance peut conduire à des erreurs d'appréciation. En dernier lieu, nous comparerons les modalités de repérage du référent de dans $x$ temps et $x$ temps plus tard.

Tout au long de l'étude, nous nous appuierons sur un corpus d'exemples puisés principalement dans une base de données contenant 230 textes (dont les plus anciens datent du $19^{\text {eme }}$ siècle) totalisant environ 22,5 millions de mots. Toutefois, lorsque nous éprouverons le besoin de confirmer notre intuition, il nous arrivera, pour disposer de données plus diversifiées, d'utiliser des exemples trouvés sur le web.

\section{Dans $x$ temps et l'indexicalité}

$\mathrm{Au}$ vu de la définition que Kleiber (1986) donne des indexicaux et que nous faisons nôtre :

«Les déictiques sont des expressions qui renvoient à un réfèrent dont l'identification est à opérer nécessairement au moyen de l'entourage spatio-temporel de leur occurrence. La spécificité du sens indexical est de "donner" le réfèrent par le truchement de ce contexte. » (Kleiber 1986 : 19)

il paraît difficile d'enrôler dans $x$ temps dans cette classe.

Il est vrai que, si l'on s'en tenait à des exemples comme (1) :

(1) - Alors, on se revoit quand?

- Demain / dans deux jours.

on pourrait être tenté de le faire, puisque la référence de demain et celle de dans deux jours se calculent par rapport au moment où sont énoncées leurs occurrences respectives. Mais l'exemple (2) montre que cette conclusion serait trop hâtive :

(2) Quand M. Dupond est parti il y a trois jours, il a dit à sa femme qu'il serait de retour dans deux jours / demain.

En effet, demain réfère, comme dans l'exemple (1), au lendemain du jour de son énonciation, alors que dans deux jours peut référer à un jour postérieur, non pas au moment de son énonciation effective, mais au moment où $\mathrm{M}$. Dupond a pris congé de sa femme en lui disant « Je serai de retour dans deux jours » (ou en employant une autre expression exprimant la

4 Dans son ouvrage de référence, Klum (1961) ne mentionne même pas dans $x$ temps parmi les expressions nynégocentriques et ne l'évoque qu'incidemment à propos de $x$ temps plus tard (1961: 267).

5 Sur l'usage que les grammairiens de l'Antiquité, et tout particulièrement Varron, faisaient de cette notion, voir Nisard (1845 : 578-579) et Douay \& Pinto (1991). 
même information). Demain est donc un authentique indexical, en ce sens que sa référence est toujours calculée par rapport au moment de son énonciation, tandis que dans $x$ temps peut être interprété par rapport au moment où il a été employé dans le cadre d'un énoncé dont on rapporte la teneur.

Mais il y a, entre demain et dans $x$ temps, d'autres différences.

Le repère par rapport auquel est interprété dans $x$ temps peut être décalé vers le futur (Berthonneau 1989 : 905) par la simple adjonction d'un indexical :

(3) [Texte daté du 13 mai 2013] - Ce n'est pas un pays qu'on associe naturellement à l'idée d'élections mais on vote pourtant en Iran et deux poids lourds, l'un libéral, l'autre conservateur, sont entrés en lice ce week-end pour le premier tour de la présidentielle qui aura lieu dans un mois demain, le 14 juin. (sur le site de l'émission Géopolitique, franceinter.fr, 13 mai 2013)

ou d'un quantième du mois qui inclut le jour de l'énonciation ${ }^{6}$ :

(4) Quand auras-tu tes quatorze ans ?

Dans trois mois le 8 . (le $8=$ le 8 de ce mois)

Une telle translation du repère ne peut pas s'appliquer aux indexicaux au sens strict et à demain en particulier ${ }^{7}$ :

(5) *Demain mardi prochain, je serai joignable toute la journée (pour référer au lendemain du jour désigné par mardi prochain)

(6) *Demain le 8 , je serai joignable toute la journée (pour référer au lendemain du 8 du mois en cours)

Néanmoins, la possibilité de translation du repère illustrée par (3) est très contrainte. Elle ne peut pas s'effectuer vers le passé :

(7) Il doit arriver *dans dix jours hier

D'autre part, dans $x$ temps est en concurrence avec dans $x$ temps d'ici, qui, lui, se comporte comme un authentique déictique. Dans l'exemple (8) :

(8) Pierre est parti ce matin à 8 heures en promettant d'être de retour dans deux heures/dans deux heures d'ici.

dans deux heures d'ici, à la différence de dans deux heures, ne peut se rapporter qu'au moment de son énonciation effective, c'est-à-dire au moment où est prononcée la phrase (8) et donc situer dans l'avenir le retour de Pierre ${ }^{8}$.

Cependant, par un aspect de son sens, dans x temps s'apparente aux indexicaux et plus particulièrement à demain : c'est ce qu'on pourrait prudemment appeler son affinité avec le futur, très clairement pointée par Henry. Que le repère soit le moment de la parole ou un moment du passé, celui-ci marque une césure entre deux formes de manifestation du temps : avant le repère, il est unilinéaire, et après lui, il présente une structure ramifiée (Gardies 1975: 51). A priori, dans $x$ temps doit donc conférer aux propositions dans lesquelles il figure une valeur non factuelle.

6 On décale le repère de façon à définir le délai exprimé par $x$ temps au moyen d'un nombre entier d'unités de temps. Ainsi, dans trois mois le 8 permet d'éviter dans trois mois et $x$ jours. Le repère décalé doit donc être défini par une unité de temps inférieure à celle utilisée dans $x$ temps. Souvent, on recourt à l'unité de temps immédiatement inférieure, par exemple le mois si le délai est exprimé en années - dans un an en avril (prochain) -, mais pas nécessairement, puisqu'on peut avoir dans un an le 8. Quoi qu'il en soit, le repère décalé se calcule par rapport au moment de l'énonciation : le 8 dans (4) dénote le 8 du mois qui inclut ce moment, et, dans dans un an en avril, en avril réfère au mois d'avril de l'année où l'on énonce cette expression.

7 Il faudrait cependant, pour dans x temps, examiner les diverses possibilités d'opérer un décalage à l'aide d'un SP introduit par à compter de (en particulier, nous semble-t-il, dans des discours de type juridique).

8 Nous ne disposons d'aucune attestation de l'emploi de dans $x$ temps d'ici dans le discours indirect, mais notre exemple construit nous paraît acceptable. 


\section{Inventaire raisonné des emplois de dans $x$ temps et demain}

Ainsi que nous l'avons dit, un tel inventaire ne peut offrir une vue exacte des emplois de dans $x$ temps et de demain que s'il tient compte des différents types de contexte dans lesquels ces expressions apparaissent.

\subsection{Les contextes : présentation}

Nous distinguerons deux types de contextes: le contexte syntaxique et le contexte discursif.

Considérons d'abord le contexte syntaxique. A côté des propositions que l'énonciateur prend en charge, il y a les propositions complétives exprimant une parole ou une pensée attribuée à un tiers. Introduites par un verbe de dire (dire, prétendre, ajouter) ou d'attitude propositionnelle (se dire, croire, penser, savoir) elles relèvent de ce qu'il est convenu d'appeler le discours indirect (dépendant), et l'on a déjà vu l'importance qu'il faut leur accorder dans l'étude des propriétés sémantiques de dans $x$ temps. Il conviendra de faire également une place au discours indirect libre. Syntaxiquement, les propositions au D.I.L. ne se distinguent pas des propositions prises en charge par l'énonciateur. Mais, d'un point de vue sémantique, il faut les traiter à part, dans la mesure où elles expriment, sur un mode particulier, les pensées ou les paroles d'un tiers.

Il est par ailleurs nécessaire de tenir le plus grand compte du contexte discursif. Il existe en effet au moins deux sortes de discours : ceux dont l'intention implicite est de décrire la réalité ou, du moins, une partie de celle-ci, et ceux qui engendrent la réalité qu'ils représentent. Ce qui les différencie, c'est leur direction d'ajustement ${ }^{9}$ : les premiers, les plus communs, s'ajustent à leur objet, alors que, dans les seconds, les récits de fiction, c'est la réalité décrite qui s'ajuste à sa description. Or, l'expression du temps dans la fiction repose sur une « grammaire » spécifique qui conditionne l'interprétation des indexicaux temporels.

On reprendra ici les analyses exposées dans Vuillaume $(1990 ; 1993)$ et notamment l'idée selon laquelle les fictions narratives s'organisent selon deux plans d'énonciation : l'un, centré sur le moment de la production du récit, à partir duquel les événements narrés sont appréhendés rétrospectivement; l'autre, objectivement fondé sur le processus de lecture, qui suscite littéralement les événements décrits et y donne accès au moment même où ils se produisent. Un exemple permettra de mieux saisir en quoi consiste cette double organisation :

(9) M. Lecoq avait une robe de chambre de velours noir à cordelière d'or et doublée de satin cerise [...] Nous savons qu'il passait la quarantaine ; mais il était conservé parfaitement et semblait être encore jeune homme $[\ldots]$

M. le marquis de Gaillardbois, plus âgé d'une dizaine d'années, était un ancien beau, fatigué, mais suffisamment confit. [...]

Au moment où nous entrons dans le sanctuaire, ces messieurs traversaient un de ces repos qui ponctuent les conversations graves, où chacun a besoin de réfléchir. (Féval, Paul, Les Habits Noirs)

Lorsqu'on analyse la phrase en italiques, on y découvre une incohérence au moins apparente. En effet, le verbe de la principale est à l'imparfait, et le procès qu'elle décrit est présenté par la quasi conjonction au moment où comme contemporain ${ }^{10}$ de celui visé par la subordonnée, qui, elle, est au présent. Comment ces deux procès peuvent-ils être présentés comme simultanés?

On pourrait être tenté d'interpréter le présent entrons comme un présent de narration, qui pourrait donc être remplacé salva veritate par une forme verbale de passé. Ici, seules deux

9 Recanati (1981: 175-181), Searle (1982: 41-42).

10 Nous employons ce terme pour de pures raisons de commodité. Pour être plus précis, il faudrait dire que le procès décrit par la subordonnée est inclus dans le laps de temps occupé par le procès visé par la principale. 
formes pourraient être envisagées, le passé simple, qui, pour des raisons qu'on n'essaiera pas d'élucider, paraît difficilement acceptable, et le passé composé :

(9a) Au moment où nous entrons/??entrâmes/sommes entrés dans le sanctuaire, ces messieurs traversaient un de ces repos qui ponctuent les conversations graves, où chacun a besoin de réfléchir.

Certes, l'emploi de la forme sommes entrés est acceptable, mais il s'accompagne d'un changement de sens. Alors que, dans le texte original, notre entrée - c'est-à-dire celle du narrateur et du lecteur - dans le sanctuaire est strictement contemporaine de la lecture de la phrase Au moment où nous... a besoin de réfléchir, le passé composé indiquerait au contraire que cette entrée a eu lieu à un moment antérieur du processus de lecture ${ }^{11}$. Il faut donc renoncer à l'hypothèse du présent de narration.

Ce qui serait tout à fait possible en revanche, c'est de remplacer l'imparfait de la principale par une forme de présent. On pourrait en effet avoir :

(9b) Au moment où nous entrons dans le sanctuaire, ces messieurs traversent un de ces repos qui ponctuent les conversations graves, où chacun a besoin de réfléchir.

Or, que fait Féval dans (9) ? Il combine tout simplement dans une seule phrase les deux perspectives : la subordonnée exprime la vision simultanée fondée sur le processus de lecture, la principale la vision rétrospective solidaire du moment de la production du récit ${ }^{12}$.

La première perspective reflète l'expérience de tout lecteur d'un texte de fiction. En quelque point du texte qu'il se trouve, sa curiosité ne concerne pas ce qui s'est passé, mais bien ce qui va se passer (Vuillaume 1990 : 55-56). Quant à la seconde perspective, elle est consubstantielle à la notion même de récit et constitue, quant à ce qui est raconté, un gage d'authenticité. Par ailleurs, ces deux configurations se distinguent par leur extension temporelle. Dans la première, le temps est borné par le présent de la lecture, au-delà duquel commence l'avenir, alors que, dans la seconde, il est borné par le moment de la production du récit, généralement conçu comme largement postérieur aux événements narrés. C'est pourquoi on peut les distinguer en qualifiant l'une de «configuration restreinte », l'autre de « configuration étendue », parce qu'elle inclut la première (Vuillaume $1990: 82$ ).

L'exemple (9) illustre un phénomène propre aux récits de fiction et qu'on qualifie communément d' $d$ intrusion d'auteur ». Ces « intrusions » mettent en scène le narrateur et le lecteur qui semblent en effet faire littéralement irruption dans l'univers narré et devenir ainsi les témoins directs des événements racontés ${ }^{13}$. Elles constituent une « fiction secondaire », qui ne peut exister sans l'autre (alors que l'inverse n'est pas vrai ${ }^{14}$ ), qu'on appellera la fiction principale. Cependant, au motif que ce phénomène se manifeste de façon sporadique et localisée, on pourrait être tenté d'en minimiser la portée, alors qu'il recèle la clé de la grammaire temporelle spécifique des textes de fiction, comme le montre l'exemple suivant :

(10) Dans le pavillon, Roland dormait enfin pour tout de bon. [...]

Il n'avait point changé : tel nous l'avons vu il y a dix ans, tel il restait sous ce rayon qui éclairait son front mâle et doux [...] Tout avait changé, cependant, autour de lui, le temps ni la mort ne s'arrêtent jamais. Il y a dix ans, celle qui passait aujourd'hui, radieuse jeune fille, dans son rêve, n'était encore qu'une enfant. Il souriait. Ses lèvres s'entrouvraient. Il songeait qu'il parlait d'amour. (Féval, Paul, Les Habits Noirs)

La proposition tel nous l'avons vu il y a dix ans fait évidemment allusion à un passage antérieur du texte dans lequel il était question de Roland, ce qui a permis au lecteur de le « voir ». Naturellement, le temps que le lecteur a mis pour lire la partie du texte qui sépare ce

11 La preuve en est fournie par l'exemple (10) infra.

12 Le constat que nous faisons ici rejoint les remarquables observations de Blin (1954: 314-319) sur les « intrusions d'auteur » et le rôle qu'y joue le processus de lecture.

13 Cf. Genette (1972 : 243-246) et Pier \& Schaeffer (2005).

14 Cette hiérarchie apparaît clairement dans les résumés de romans : jamais les événements de la fiction secondaire n'y sont évoqués. 
passage de celui qu'il est en train de lire ne saurait être objectivement de dix ans. Mais, comme la durée de ce processus est essentiellement variable et ne peut être mesurée, elle est conventionnellement assimilée à la durée des événements dont le lecteur a pris connaissance depuis le premier passage consacré à Roland. Et on notera que, dans il y a dix ans, le verbe avoir est au présent, parce que le repère est le moment où on lit cette expression (le présent ne pourrait pas être remplacé par un imparfait).

Considérons maintenant la phrase Il y a dix ans, celle qui passait aujourd'hui, radieuse jeune fille, dans son rêve, n'était encore qu'une enfant. On y trouve une nouvelle occurrence de l'expression il y a dix ans, qui est justiciable de la même explication que la première. Mais, ici, elle est mise en contraste avec aujourd'hui, ce qui ne serait pas possible si les deux expressions relevaient de deux systèmes de repérage temporel différents et prouve qu'aujourd'hui est lui aussi relatif au processus de lecture et désigne donc le jour où on lit ce mot $^{15}$ - ce jour étant, en vertu de la convention signalée supra, inscrit dans le calendrier de l'univers narré, comme l'atteste l'exemple suivant :

(11) Nous entrons à l'hôtel de Clare dans la soirée du mardi 3 janvier 1843, à peu près à l'heure où les anciens clercs de l'étude Deban, le roi Comayrol en tête, quittaient le salon du bon Jaffret pour rentrer à leurs domiciles respectifs [...] (Féval, Paul, Les Habits Noirs)

où les faits et gestes du narrateur et du lecteur - qui se déplacent dans l'espace de l'univers narré - sont inscrits dans le même calendrier que celui dans lequel s'ordonnent les événements du récit.

Si les «intrusions d'auteur» sont devenues très rares dans la littérature française contemporaine $^{16}$, en revanche, les cooccurrences d'indexicaux temporels comme aujourd'hui ou maintenant avec des formes verbales de passé sont restées très courantes.

Nous allons voir maintenant plus précisément comment contexte syntaxique et contexte discursif retentissent sur l'emploi de dans $x$ temps et demain.

\subsection{Les contextes : illustrations}

\subsubsection{Le discours indirect dépendant}

Revenons à la comparaison des comportements respectifs de demain et dans $x$ temps dans les complétives du discours indirect, et, plus précisément, à l'exemple (2). Dans le cadre d'un échange verbal ordinaire ou, plus généralement, dans un discours véridique, demain employé dans une complétive de discours rapporté ne peut référer qu'au lendemain du jour de son énonciation effective, alors que dans $x$ temps peut être interprété par rapport au moment où a été énoncé le propos dont le contenu est restitué par la complétive (cf. notre analyse de l'exemple (2)). Considérons maintenant, pour le cas de la fiction, les deux exemples suivants :

(12) Une fois dans la rue, Docile tourna rapidement la manivelle qui faisait descendre le rideau de fer. Celui-ci tomba presque d'un coup, en grinçant. Elle se dit que demain, si l'homme ne l'avait pas esquintée au point qu'elle soit incapable de venir travailler, elle irait dans un garage acheter de la graisse [...]. (Decoin, Didier, Docile)

(13) Un jour, enfin, Zagouliaïeff annonça que les renseignements recueillis étaient suffisants et que l'attentat aurait lieu dans quarante-huit heures. (Troyat, Henri, Tant que la terre durera, 792)

En (13), la référence de dans quarante-huit heures est calculée à partir du moment où Zagouliaïeff a fait son annonce, c'est-à-dire de la même façon que dans deux jours dans l'exemple (2). Mais, comme on l'a dit supra, si on appliquait la même interprétation à demain dans (12), il faudrait encore expliquer pourquoi une telle interprétation n'est pas possible dans un discours véridique. En fait, la solution est à portée de la main : comme, dans le discours rapporté, la référence des indexicaux est calculée par rapport au moment de l'énonciation

15 Ce qui prouve donc également que les combinaisons «aujourd'hui + temps du passé », très courantes dans les textes de fiction, sont des manifestations du même phénomène que (10).

16 A la notable exception de l'œuvre de Jean Echenoz. 
intégrante, celle de demain est identifiée ici, exactement comme aujourd'hui dans l'exemple (10), relativement au jour où on lit ce mot, qui est aussi celui où Docile constate que le rideau de fer de son magasin a besoin d'un graissage. Mais, comme l'activation de la configuration restreinte fait du lecteur le témoin direct des événements racontés, il a le sentiment d'être le contemporain de Docile, et, grâce à l'omniscience du narrateur, de lire à livre ouvert dans ses pensées $^{17}$ - impression que ne produit évidemment pas l'exemple (10), qui ne relève pas du discours rapporté.

La différence entre le mode de calcul de la référence de dans quarante-huit heures et celui qui s'applique à demain n'est guère perceptible, mais ceci s'explique aisément. En effet, le présent fondé sur le processus de lecture dans l'exemple (12) est, avons-nous dit, le jour où le rideau de fer du magasin de Docile se détraque, mais ce jour est également celui où elle réfléchit à ce qu'elle doit faire pour remédier à ce problème. Inversement, la référence de dans quarante-huit heures dans (13) est évaluée à partir du moment où Zagouliaïeff annonce l'attentat (un jour), mais ce pourrait être aussi bien le moment correspondant au présent déterminé par le processus de lecture.

Aussi subtile soit-elle, cette différence entre demain et dans quarante-huit heures ne peut être négligée si on veut rendre compte du fait que (13) pourrait figurer dans un récit véridique (i.e. avec un fonctionnement tout à fait ordinaire de dans quarante-huit heures), alors que la phrase «Elle se dit que demain... » ne le pourrait pas.

Il reste à envisager le cas particulier de discours indirect où la complétive est enchâssée sous un verbe d'attitude propositionnelle de type factif (savoir, se douter, ignorer, etc.), présupposant la vérité de la proposition introduite, et la connaissance de celle-ci par l'énonciateur ${ }^{18}$. C'est le cas, pour un discours véridique, dans l'exemple ci-dessous :

(14) 11 Novembre 1918 il y a 100 ans

Message par Tranter 06 Nov 2018 11:24

Il y a cent ans mes deux Grands Pères ne se doutaient pas que dans quelques jours cette guerre $s$ 'arrêterait et qu'ils allaient pouvoir rentrer

Ils en sont revenus tous les deux, un peu amochés et traumatisés par ce qu'ils ont vécus, mais vivants (tirmaillyforum.com, « Forum des tireurs à la cible à longue distance »)

Le SP dans quelques jours est ici interprété par rapport au moment où le sujet du verbe d'attitude propositionnelle (mes deux Grands Pères) est saisi - c'est-à-dire cent ans jour pour jour avant l'énonciation de (14), le 6 novembre 1918, à une date où l'armistice du 11 novembre appartenait encore à l'avenir et ne pouvait donc être que conjecturé ${ }^{19}$. À cette perspective prospective, commune à tous les cas de discours indirect, s'ajoute cependant la perspective rétrospective de l'énonciateur à qui est attribué - du fait du caractère factif de $s e$ douter - la connaissance de ce qui s'est effectivement passé. C'est ainsi la combinaison de deux perspectives temporelles distinctes qui explique que dans $x$ temps puisse, dans une telle configuration avec verbe factif, être associé à une proposition relevant d'une interprétation factuelle (ce qui n'était pas le cas pour dans deux jours en (2)). On verra infra qu'il existe d'autres contextes permettant la coexistence d'une visée prospective et d'une visée rétrospective.

Passons maintenant à la comparaison de dans x temps et de demain. Dans la mesure où l'exemple (14) relève d'un discours véridique, on constate sans surprise que l'emploi de demain n'y serait pas possible. Pour le vérifier, on supposera que la date du message est, non pas le 6, mais le 10 novembre 2018 :

$17 C f$. Vuillaume $1990: 107$.

18 Les propriétés présuppositionnelles (et autres que présuppositionnelles) des verbes factifs sont détaillées par Kiparsky \& Kiparsky (1971).

19 Que le sujet de pensée en question, en l'occurrence les deux grands-pères, fasse ou non cette conjecture ne change rien à l'affaire : ce dont il s'agit en effet, c'est uniquement d'une possibilité de pensée, de croyance. 
(14a) Il y a cent ans mes deux Grands Pères ne se doutaient pas que dans quelques heures/*demain cette guerre s'arrêterait.

Le constat donc est ici le même que celui que nous avons fait à propos de la complétive de discours indirect dans l'exemple (2).

Si on considère en revanche les phrases à verbe factif dans l'environnement discursif d'une fiction, les conditions changent, et demain (exemple (15)) peut alors s'employer aussi bien que dans $x$ temps (exemple (16)) - quoiqu'avec les mêmes différences, dans le mode de calcul de la référence, que celles qu'on pointait plus haut entre les exemples (12) et (13) ${ }^{20}$ :

(15) Avec quelle tranquillité atroce Marie Ossipovna prononçait les mots de «paralysie », de « mort », de « râle », et le nom même du défunt ! Était-ce un signe de courage, ou d'indifférence ? Savaitelle qu'elle était veuve [...] ? Savait-elle que sa vraie solitude commencerait demain, lorsque les dernières pelletées de terre auraient enseveli le cercueil aux poignées d'argent ? (Troyat, Henri, Tant que la terre durera)

(16) Pour l'heure, j'étais à Étretat. Un collectionneur d'armes du bassin d'Arcachon devait astiquer ses plus belles pièces sans se douter que j'allais, dans quelques jours, faire main basse sur tout son arsenal. (Commère, Hervé, J'attraperai ta mort)

\subsubsection{Le discours indirect libre}

En ce qui concerne le D.I.L., la comparaison entre les deux contextes discursifs (discours véridiques $v s$. fictions) pose un problème pratique presque insoluble. Il semble totalement absent des ouvrages historiques contemporains. Il est en revanche possible dans les échanges verbaux ordinaires, mais ne s'y manifeste que dans des fragments très brefs, de sorte qu'il est extrêmement difficile d'y trouver des exemples adaptés à notre propos. Nous risquerons donc le suivant, tout droit sorti de nos ateliers :

(17) J'ai rencontré Pierre avant-hier. Je l'ai trouvé d'excellente humeur. Pour une fois, il ne parlait pas de son travail, mais de ses vacances. Il partait dans deux-jours/demain pour Tahiti...

Alors que dans deux jours est évalué par rapport au moment où Pierre parlait de ses projets, demain ne peut se rapporter qu'au moment de son énonciation effective.

Dans les textes de fiction, en revanche, et pour les mêmes raisons que celles déjà données au sujet du discours indirect dépendant à propos des exemples (12) et (13), demain et dans $x$ temps s'emploient l'un aussi bien que l'autre (avec, là encore, le même type de différence dans le calcul de la référence) :

(18) On s'accorda donc pour faire d'abord le guet. On verrait si Georges Gerfaut rentrait. Sinon, à la nuit tombée, on pénétrerait dans son appartement par acquit de conscience. Et s'il n'était pas là, on enverrait un télégramme bidon à Saint-Georges, pour dire à l'homme qu'il y avait une fuite d'eau chez lui, et qu'il veuille bien appeler son domicile aussi vite que possible. On passerait la nuit dans l'appartement. [...]

Bref, demain matin, s'il était avéré que Gerfaut était retourné à Saint-Georges-de-Didonne, on aviserait, on retournerait sans doute là-bas et l'on abattrait l'homme, vraisemblablement au fusil. (Manchette, Jean-Patrick, Le petit bleu de la côte Ouest)

(19) Vallorge, lui, édifiait ses plans à très lointaines échéances. Suraisne comptait remplacer le vieux titulaire de la chaire d'anatomie dès que celui-ci mourrait, ce qui ne tarderait guère. [...] Dans le sillage de Suraisne, Vallorge deviendrait d'abord agrégé, puis professeur d'anatomie. Et quand Suraisne, dans cinq ans, succéderait à Ribières aux voies respiratoires, Vallorge postulerait la chaire d'anatomie devenue vacante. (Van der Meersch, Maxence, Corps et âmes)

\subsubsection{Paroles de narrateur}

Les faits analysés jusqu'ici relevaient du discours rapporté, mais notre corpus contient aussi des exemples où dans $x$ temps et demain apparaissent dans des phrases dont on ne peut attribuer la prise en charge qu'au narrateur, et, comme on va le voir, ces phrases ne sont pas

20 Par ailleurs, même s'il s'agit de fiction, l'emploi d'un verbe factif amène dans tous les cas à regarder l'énonciateur comme « garant» de la valeur factuelle de la complétive. 
interprétées exactement de la même façon selon qu'elles figurent dans des narrations en $3^{\text {ème }}$ personne ou dans des récits autobiographiques.

\subsubsection{Dans les narrations en $3^{\text {ime }}$ personne}

Voici deux exemples empruntés à des récits en $3^{\text {ème }}$ personne :

(20) Jean Oullier avait entendu le signal [...] il fit brusquement demi-tour, et, au lieu de continuer sa route vers l'escarpement et le marais, il descendit rapidement la colline. Il ne courait plus, il volait! Sans doute avait-il trouvé quelque plan qu'il avait hâte de mettre à exécution.

$\mathrm{Au}$ reste, du train dont il allait, dans quelques minutes il aurait rejoint ses amis. (Dumas, Alexandre, Les Louves de Machecoul)

(21) M. de Villefort n'avait point revu le vieillard depuis le matin de cette mort. Toute la maison avait été renouvelée : $[\ldots]$ : tous, jusqu'au concierge et au cocher, offraient de nouveaux visages qui s'étaient dressés pour ainsi dire entre les différents maîtres de cette maison maudite et avaient intercepté les relations déjà assez froides qui existaient entre eux. D'ailleurs les assises s'ouvraient dans trois jours, et Villefort, enfermé dans son cabinet, poursuivait avec une fiévreuse activité la procédure entamée contre l'assassin de Caderousse. (Dumas, Alexandre, Le Comte de Monte Cristo)

Ils ne présentent aucune ambiguïté, en ce sens qu'on ne peut pas les interpréter comme relevant du discours indirect libre et que c'est donc nécessairement le narrateur qui s'y exprime. En un certain sens, ils constituent une curiosité narrative. Certes, le narrateur peut très légitimement se livrer à des anticipations, c'est-à-dire effectuer une sorte de saut dans le temps pour évoquer des événements postérieurs à ceux qu'il est en train de raconter, mais qui se sont effectivement produits ${ }^{21}$. Cependant, ce dont il est question ici, c'est autre chose : ce sont des événements conçus comme futurs et dont la réalisation, certes probable, n'est pas garantie. Et, de fait, il serait impropre de dire que le narrateur nous les raconte.

Pour que celui-ci puisse évoquer un événement dans une perspective future, il faut qu'il borne son horizon temporel au présent défini par le processus de lecture, présent qui correspond, dans la fiction principale, au dernier événement effectivement raconté. En conséquence, ce qui est relégué dans le passé par les morphèmes verbaux (aurait dans (20), s'ouvraient dans (21)), ce n'est pas, dans un cas comme dans l'autre, l'événement décrit, mais la conjecture dont il est l'objet.

Nous examinerons encore un autre exemple, du même type, mais incluant un emploi très intéressant du verbe savoir :

(22) [Pour interpréter l'exemple ci-dessous, il faut préciser que Horatio Patterson accompagne, pour un voyage à destination des Antilles, les élèves d'un collège londonien qu'on a récompensés de leur travail par l'octroi de bourses qui doivent leur permettre de rendre visite à leurs familles. Or, avant leur embarquement, des bandits conduits par Harry Markel ont assassiné tout l'équipage et, pour ne pas donner l'éveil avant d'avoir pris la mer, se conduisent comme les membres d'un équipage ordinaire, à l'instar de Wagah qui joue le rôle de steward. Le projet de la bande est de tuer tous les passagers dès que le navire sera au large.]

[...] par sa minutie dans le service, par le zèle qu'il affectait, Wagah devait plaire à un homme, on peut le dire, aussi naïf que l'économe d'Antilian School. Harry Markel avait eu la main heureuse en le choisissant pour ces fonctions de steward. Personne n'avait mieux joué son rôle. Eût-il eu à le continuer pendant toute la traversée que M. Horatio Patterson n'aurait jamais soupçonné ce misérable. Or, on ne le sait que trop, ce rôle allait prendre fin dans quelques heures. (Verne, Bourses de voyage)

Pour comprendre le problème d'interprétation posé par la dernière phrase de cet extrait, il faut savoir que la mise à exécution du projet d'assassinat sera finalement différée sur décision de Harry Markel. La seule différence entre cet exemple et les deux exemples

21 Comme dans :

«On était, comme nous l'avons dit, au 15 août. Le soleil tombait d'aplomb sur les épaules des dieux de marbre et de bronze ; il chauffait l'eau des conques et mûrissait dans les vergers ces magnifiques pêches que le roi devait regretter cinquante ans plus tard, [...]» (Dumas, Alexandre, Le Vicomte de Bragelonne) 
précédents, c'est que la prévision exprimée par ce rôle allait prendre fin dans quelques heures est explicitement démentie par la suite des événements. Mais il faut aussi tenir compte de l'incise on ne le sait que trop.

Si savoir était à l'imparfait (on ne le savait que trop), son sujet, on, ne pourrait référer qu'à des personnages du récit et, parmi ceux-ci, seulement à Harry Merkel et sa bande. Mais, dans ce cas, la présence de ne...que trop ne se justifierait pas, puisque les bandits ne pensent pas avoir quoi que ce soit à redouter de l'avenir. En outre, en employant le verbe savoir, le narrateur nous induirait délibérément en erreur en se portant garant de la vérité de ce rôle allait prendre fin dans quelques heures, alors qu'il sait, lui, au moment où il raconte l'histoire, que la mise à exécution du projet d'assassinat sera remise à plus tard. En employant savoir au présent, il évite cet écueil. Il se positionne en quelque sorte à côté du lecteur, dans le présent du processus de lecture, et limite ainsi son savoir à cet horizon temporel. Ce faisant, il se prémunit contre le reproche qui pourrait lui être adressé d'avoir délibérément trompé ses lecteurs. Il évite simplement de leur révéler trop tôt le coup de théâtre qui se prépare. En somme, il ménage ses effets ${ }^{22}$.

Si notre corpus contient d'autres exemples de dans $x$ temps semblables à ceux que nous venons de citer, il ne comporte en revanche qu'un seul emploi analogue de demain:

(23) [Cet extrait figure au tout début d'un chapitre du Bossu intitulé "Le plaidoyer ». La dernière scène du chapitre précédent s'est déroulée dans l'oratoire de la princesse de Gonzague. Celle-ci vient de découvrir dans son livre d'heures un mot déposé par une main anonyme et l'exhortant à se présenter au " tribunal de famille » qui doit se tenir dans la grande salle de l'hôtel de Lorraine. La princesse n'a pas été témoin de ce qui vient de se passer dans cette salle, et, comme elle vit en recluse, elle n'est certainement pas au courant de ce qui doit s'y passer le lendemain. On ne peut donc pas interpréter la phrase "Cette grande salle de l'hôtel de Lorraine devait être demain polluée..." comme relevant du discours indirect libre. Elle exprime un simple projet, valide le jour où doit se tenir le " tribunal de famille », mais dont rien ne dit qu'il se réalisera.]

Cette grande salle de l'hôtel de Lorraine, qui avait été déshonorée ce matin par l'ignoble enchère, qui demain devait être polluée par le troupeau des brocanteurs adjudicataires, semblait jeter à cette heure son dernier et brillant éclat. Jamais, assurément, fût-ce au temps des grands ducs de Guise, assemblée plus illustre n'avait siégé sous sa voûte. (Féval, Paul, Le Bossu)

Nous ne le commenterons pas en détail, étant donné qu'il est en tout point semblable aux exemples (20)-(21).

\subsubsection{Dans les récits en $1^{\text {ir }}$ personne (ou autobiographiques)}

Examinons maintenant d'autres exemples qui ne diffèrent des précédents que par leur contexte discursif :

(24) Mon arrivée au palais Pitti ? Mes premières impressions ? Quelle demeure sévère ! Quelle bâtisse sans charme ! Pour des enfants, grandir dans cette prison! Et quelle surprise - la première d'une longue suite d'étonnements éprouvés dans l'intimité de cette famille - lorsque le grand-duc, suivi de son fils aîné et de sa fille, l'un aimable et volubile, l'autre hautaine et renfrognée, me fit, dès le lendemain, visiter une partie des appartements d'apparat - ceux-là mêmes qui deviendraient dans

22 Notons que l'analyse ne serait pas très différente si, au lieu d'une incise, il s'agissait d'un discours indirect introduit par le verbe factif savoir ("Or, on ne sait que trop que ce rôle allait prendre fin dans quelques heures ») - ce qui nous ramènerait à la configuration traitée plus haut à propos de l'exemple (16). Dans ce type de cas cependant, où l'énonciateur ne se distingue pas nettement du sujet du verbe factif (le on incluant l'énonciateur et son lecteur), il n'y a pas de double perspective temporelle, prospective et rétrospective, comme on l'avait vu pour l'exemple (14). Il apparaît ainsi que l'emploi d'un verbe factif n'empêche pas qu'un suspens puisse continuer d'être ménagé, à condition que le sujet en soit, non pas un personnage, mais l'énonciateur même. On citera encore en ce sens, comme exemple attesté, l'extrait suivant de Jules Verne : «On sait, en outre, qu'une tante d'Amasia [...] lui avait légué en mourant l'énorme somme de cent mille livres, à la condition qu'elle fût mariée avant seize ans révolus [...] et l'on sait que ce délai expirait dans six semaines. » (On a en effet appris cette condition au chapitre III) (Verne, Jules, Kéraban-le-têtu). 
quelques années, sous le nom de Galerie Palatine, le domaine réservé de Giovan Gastone. (Fernandez, Dominique, Le Dernier des Médicis)

Le premier constat qui s'impose, c'est que la relative est comprise comme dénotant un événement qui s'est réellement produit. Mais qu'est-ce qui conduit à cette interprétation ? Il y a au moins deux facteurs, qui peuvent passer inaperçus, mais qui n'en jouent pas moins un rôle déterminant. Au premier chef, la séquence ceux-là mêmes ... qui invite le lecteur à reconnaître l'identité de deux entités qu'il aurait pu croire distinctes: d'une part, les appartements d'apparat dont le narrateur relate la visite qu'il a été invité à y faire et, d'autre part, la mention de la Galerie Palatine - qui est le deuxième élément en jeu -, dont tout le monde, ou, à tout le moins, les lecteurs du Dernier des Médicis, connaît au moins le nom. Il est donc fait appel aux connaissances du lecteur, qui, lui, appréhende les événements narrés rétrospectivement, et donc à la lumière de ses connaissances, et c'est ce qui conduit à l'interprétation factuelle. Du reste, si on supprimait ceux-là mêmes et sous le nom de Galerie Palatine :

(24a) Le duc me fit, dès le lendemain, visiter une partie des appartements d'apparat, qui deviendraient dans quelques années le domaine réservé de Giovan Gastone.

on interprèterait la relative comme l'expression d'un projet dont le visiteur/narrateur a eu vent d'une façon ou d'une autre, mais dont la réalisation resterait à confirmer. Il s'agirait, dans ce cas, de style indirect libre, comme le prouve la possibilité d'ajouter une incise dans la relative :

(24b) Le duc me fit, dès le lendemain, visiter une partie des appartements d'apparat, qui deviendraient dans quelques années, m'avait-on dit, le domaine réservé de Giovan Gastone.

On a donc, dans l'exemple (24), la combinaison de deux visées, l'une prospective, celle $\mathrm{du}$ visiteur du palais Pitti, l'autre rétrospective, celle du lecteur, mais aussi du narrateur, puisque celui-ci a été le témoin de la transformation des appartements d'apparat en domaine réservé de Giovan Gastone. Et le rapport entre ces deux perspectives rappelle celui qui existe, dans les exemples à verbe factif, entre le point de vue du sujet de l'énonciation et celui du sujet de l'énoncé.

Soit encore l'exemple suivant :

(25) Et M. de Charlus ne se contentait même pas d'omettre dans la conversation Mme Verdurin [...] ; il faisait même des critiques sur toute la partie de la soirée dont Mme Verdurin était responsable : « Mais, à propos de tasse, qu'est-ce que c'est que ces étranges demi-bols, pareils à ceux où, quand j'étais jeune homme, on faisait venir des sorbets de chez Poiré Blanche ? Quelqu'un m'a dit tout à l'heure que c'était pour du “café glacé". Mais en fait de café glacé, je n'ai vu ni café ni glace. Quelles curieuses petites choses à destination mal définie ! » Pour dire cela, M. de Charlus avait placé verticalement sur sa bouche ses mains gantées de blanc et arrondi prudemment son regard désignateur, comme s'il craignait d'être entendu et même vu des maîtres de maison. Mais ce n'était qu'une feinte, car dans quelques instants il allait dire les mêmes critiques à la Patronne elle-même, et un peu plus tard lui enjoindre insolemment. «Et surtout plus de tasses à café glacé ! Donnez-les à celle de vos amies dont vous désirez enlaidir la maison. [...]» (Proust, Marcel, A la recherche du temps perdu)

Comme dans (24), on a ici l'expression de deux points de vue, celui du narrateur narré (intradiégétique) et celui du narrateur narrant (extradiégétique). Le premier - même s'il connaît bien la personnalité et les manières de $\mathrm{M}$. de Charlus, même si, témoin des précautions que celui-ci feint de prendre pour n'être pas entendu, il se doute que quelque chose se prépare - n'est pas en mesure de prévoir la déclaration provoquante que va faire $\mathrm{M}$. de Charlus. Seul le narrateur narrant est en mesure de la citer littéralement.

La différence entre les exemples (20)-(21), d'une part, et (24)-(25), d'autre part, est évidente. Dans les seconds, la perspective conjecturale se double d'une vision rétrospective qui confère à la proposition une valeur factuelle. Dans ce type de configuration en effet, le narrateur se dédouble en deux instances : le narrateur narrant (extradiégétique) et le narrateur narré (intradiégétique). Pour le second, les faits s'accomplissent et se révèlent au même 
rythme que pour le lecteur qui les découvre en lisant, alors que, pour le premier, ils appartiennent à un passé dont il connaît tous les détails. Alors que, dans les récits en $3^{\text {ème }}$ personne, le narrateur narrant peut se faire oublier en bornant son propre horizon temporel au présent du processus de lecture ${ }^{23}$, dans les récits en $1^{\text {ère }}$ personne, les deux modes de manifestation du narrateur sont indissociables : le narrateur narrant ne peut se faire oublier et il ne peut pas davantage faire oublier que tout ce qui est narré lui est déjà connu ${ }^{24}$.

\subsection{Les emplois rebelles}

Outre les exemples dont nous avons essayé de rendre compte supra, nous en avons trouvé un certain nombre qu'il n'est pas possible d'expliquer au moyen des concepts dont nous sommes servis jusqu'ici. Ces exemples font tous partie de discours véridiques en $3^{\text {ème }}$ personne. En voici deux qui nous permettront de mieux cerner la difficulté :

(26) Ce fut à la table de Laurent de Médicis que le Florentin Louis Pulci lut, pour la première fois, son poème de Morgant-le-Géant (Morgante-il-Maggiore). C'est le genre de poésie qui devait dans quelques années immortaliser le nom de l'Arioste. (Duquesnel, Amédée, Histoire des lettres avant le christianisme: cours de littérature, Volume 4 - texte accessible sur books.google.fr)

(27) A Grenoble, la «Propagation de la Foi » fut fondée le 8 février 1647, dans l'hôtel de M. Jean de Chevrières, alors président au Parlement de Bourgogne, et qui devait, dans quelques années, passer au Parlement de Dauphiné, et devenir un des confrères les plus actifs « du SaintSacrement ». Elle décidait, ce jour-là, d'être dans la dépendance directe de celle de Paris. Le dimanche 17 février, elle était définitivement constituée et nommait ses officiers. (Allier, Raoul, La Cabale des Dévots - texte accessible sur books.google.fr)

On le voit immédiatement: les propositions concernées ne relèvent ni du discours indirect dépendant, ni du discours indirect libre. D'autre part, comme il s'agit de textes historiques (donc de discours véridiques), on ne peut invoquer l'existence de deux plans d'énonciation. Enfin, dans nos deux exemples, dans $x$ temps est combiné avec la périphrase devait + infinitif, qui, en français moderne, sert notamment à l'expression du prospectif, c'està-dire à l'évocation anticipée d'événements qui se sont réellement produits (cf. supra). On parle alors d'emploi "objectif», emploi auquel se prêtent également le conditionnel et la périphrase allait + infinitif. C'est également de cet emploi qu'il s'agit ici. Seulement, il n'est, aujourd'hui, compatible qu'avec $x$ temps plus tard, mais pas avec dans $x$ temps.

La solution du problème nous semble être d'ordre diachronique. L'émergence de l'interprétation objective du conditionnel et des périphrases allait/devait + infinitif date du début du $\mathrm{XIX}^{\mathrm{e}}$ siècle. Jusque-là, ces formes verbales ne connaissaient que des emplois subjectifs - c'est-à-dire cantonnés au discours indirect ou à des contextes apparentés - où elles pouvaient se combiner avec dans $x$ temps. Nous faisons donc l'hypothèse que, lors de

23 Et, s'il ne le fait pas, alors la valeur conjecturale disparait, et ce n'est plus dans $x$ temps qu'il emploie, mais $x$ temps plus tard.

24 On trouve, en cherchant bien, des configurations où le narrateur d'une narration en $3{ }^{\text {ème }}$ personne fait part de ce qu'il sait devoir advenir en se plaçant dans une perspective prospective (avec emploi de dans $x$ temps, donc, plutôt que de $x$ temps plus tard). Ce peut être le cas dans des fictions où un personnage est amené à retourner dans le passé (avec toute la connaissance qu'il a de ce qui, à partir de ce passé, apparaît comme un futur). Le narrateur, dans ce cas, fait corps avec son personnage pour regarder avec lui, dans une vision prospective, ce qu'il sait s'être effectivement passé dans ce futur. On mentionnera par exemple :

«Un autre personnage sortit de l'ombre. Un homme d'âge moyen, à la barbe claire et au regard métallique. Vincent retint son souffle : il avait reconnu le docteur Sherman, qui serait dans quelques années le médecin personnel de Joseph Poney ». (Jeury, Michel, Poney-Dragon - texte accessible sur books.google.fr)

Tout particulier qu'il soit, cet exemple continue de mettre en évidence la précision des rouages dans le fonctionnement de l'expression dans $x$ temps : utilisée avec une visée prospective dans une narration en $3^{\text {ème }}$ personne où elle n'aurait «normalement » pas sa place, elle trahit le bouleversement des règles temporelles ordinaires, selon lesquelles il est impossible d'avoir vécu l'avenir avant le passé. 
l'émergence de leur sens objectif ${ }^{25}$, le conditionnel et les deux périphrases allait/devait + infinitif sont restées, pendant une période de transition, solidaires de leur partenaire adverbial habituel dans $x$ temps, lequel n'a été évincé par $x$ temps plus tard que dans un deuxième temps ${ }^{26}$. Le fait que tous les exemples que nous avons recensés datent du XIX ${ }^{\mathrm{e}}$ siècle nous conforte dans cette idée.

\section{$3 \quad$ Quels repères pour dans $x$ temps et $x$ temps plus tard ?}

\subsection{X temps plus tard}

Le statut anaphorique de $x$ temps plus tard, qui fait largement consensus, a plusieurs conséquences :

1. La première - triviale -, c'est que l'emploi de $x$ temps plus tard n'est possible qu'à condition que le contexte discursif amont i) permette d'accéder à un repère, et ii) que celuici soit connu avec un degré de précision égal à l'unité de temps utilisée pour spécifier le laps de temps défini par $x$ temps. Ainsi, c'est l'absence de repère contextuel qui interdit la substitution de vingt minutes plus tard à dans vingt minutes dans :

(28) Elle [= la marquise] sonna et quand le domestique fut entré, comme elle ne dissimulait nullement et même aimait à montrer que son vieil ami passait la plus grande partie de son temps chez elle : - Allez donc dire à M. de Norpois de venir, il est en train de classer des papiers dans mon bureau, il a dit qu'il viendrait dans vingt minutes/*vingt minutes plus tard et voilà une heure trois quarts que je l'attends. (Proust, Marcel, A la recherche du temps perdu)

2. La seconde conséquence, c'est que $x$ temps plus tard permet d'inscrire l'événement qu'il date dans la continuité des événements racontés, alors que dans $x$ temps ne le peut pas, ou seulement de façon indirecte. Dans l'exemple ci-dessous, l'expression quelques heures plus tard, qui figure dans le texte original, pourrait être remplacée par dans quelques heures:

(29) La nuit était noire. La lune, nouvelle ce jour-là même, avait disparu en même temps que le soleil. Ainsi que l'avait fait observer Harbert, de gros nuages orageux formaient une voûte basse et lourde, qui empêchait tout rayonnement d'étoiles. Quelques éclairs de chaleur, reflets d'un orage lointain, illuminaient l'horizon. [554|555] Il était possible que, quelques heures plus tard/dans quelques heures, la foudre tonnât sur l'île même. C'était une nuit menaçante. (Verne, Jules, L'île mystérieuse)

mais au prix d'un changement de sens. Quelques heures plus tard, en vertu de ses propriétés anaphoriques, récupère les informations contenues dans la phrase précédente. Le moment où la foudre tonnera peut-être sur l'île est donc calculé à partir de celui où les éclairs de chaleur illuminent l'horizon, ce qui souligne la continuité entre les deux événements. Avec dans quelques heures, rien ne l'indiquerait. On comprendrait en effet que la phrase Il était possible ... l'île même est l'écho au discours indirect libre des réflexions que se font les voyageurs («Il est possible que, dans quelques heures, la foudre tonne sur l'île même »). Dans ce cas, le référent de dans quelques heures serait calculé à partir du moment de l'emploi de cette expression, et ce moment serait lui-même situé dans le cours du récit en fonction du dernier événement évoqué (les éclairs de chaleur illuminant l'horizon). Bref, le lien entre les différents événements serait perdu.

3. La troisième conséquence du statut anaphorique de $x$ temps plus tard, c'est qu'il ne permet pas, par lui-même, de situer son référent par rapport à la partition passé / présent / futur, puisque tout dépend du site temporel de l'antécédent. En toute rigueur, il serait préférable de le considérer comme une expression chronologique plutôt que comme une expression

25 Cf. Nilsson-Ehle (1943/44).

26 Certaines cooccurrences de dans $x$ temps avec les périphrases allait/devait + infinitif en français contemporain posent des problèmes que, faute de place, nous renonçons à traiter ici. 
temporelle. À l'inverse, comme on va le voir ci-après, dans $x$ temps est évalué par rapport à un repère qui situe automatiquement son référent dans le temps proprement dit.

\subsection{Dans $x$ temps}

Le repère par rapport auquel est évalué dans $x$ temps est de type énonciatif. Cette formulation peut paraître curieuse, mais elle répond à une nécessité. On ne peut pas dire en effet, sans autre précision, que la référence de dans $x$ temps est toujours évaluée par rapport au moment de son énonciation. Rappelons en effet - bien que ce soit une banalité - que l'énonciation d'un signe est un événement qui s'accomplit et se manifeste sans être décrit, mais que, par exemple dans le discours indirect dépendant, la référence de dans $x$ temps peut être calculée, non pas par rapport à son énonciation effective, mais par rapport au moment où ce SP a été (ou sera) employé dans un énoncé dont on restitue la teneur. Dans ce cas, l'énonciation pertinente pour l'interprétation de dans $x$ temps est décrite/représentée (et non manifestée) par la proposition dont le verbe régissant est la tête.

Le repère est une énonciation représentée lorsque dans $x$ temps apparait dans une proposition régie par un verbe introducteur de discours indirect (qu'il s'agisse ou non d'un verbe factif).

Dans les autres cas, dans $x$ temps est interprété par rapport au moment de son énonciation effective. C'est évident lorsqu'il apparaît, dans le cadre d'un échange verbal ordinaire, dans une proposition directement prise en charge par le locuteur - cf. exemple (1). Mais c'est aussi le cas dans les phrases que nous avons examinées sous 2.2.3.1. Dans l'exemple (21), la phrase les assises s'ouvraient dans trois jours n'a de sens que relativement à un moment où l'ouverture des assises appartenait encore à l'avenir. Il s'ensuit que le repère auquel se rapporte dans trois jours ne peut être que le présent (faute de quoi dans trois jours ne pourrait référer à l'avenir) déterminé par le processus de lecture, et que la marque d'imparfait ne sert pas à situer dans le passé l'événement lui-même, mais la conjecture dont il est l'objet.

Mentionnons enfin le cas particulier de dans $x$ temps demain ou dans trois mois le $8 . \mathrm{Si}$ l'on considère dans $x$ temps isolément, alors il y a lieu, comme nous l'avons fait supra de considérer qu'il y a ici un décalage du repère. Mais si l'on envisage la jonction en bloc, on peut la considérer comme un indexical, puisque le calcul de sa référence s'effectue bel bien à partir du moment de son énonciation et que le calcul du laps de temps qui sépare le référent du repère est le résultat d'une simple addition ${ }^{27}$. Cette option semble d'autant plus légitime que le choix du second terme de la construction est extrêmement contraint (cf. note 6).

\subsection{La spécificité de la référence des indexicaux temporels}

Dans les travaux de linguistique - mais plus encore dans les recherches en philosophie du langage - qui traitent des indexicaux temporels, on a coutume de définir ceux-ci au moyen d'expressions réputées « objectives », c'est-à-dire par une date du calendrier complétée, le cas échéant, par une indication horaire. Si le procédé est légitime, il risque cependant d'accréditer l'idée que, si la phrase :

(30) Aujourd'hui, il fait un temps magnifique.

est énoncée le 14 juillet 2019, cette date est le référent de l'occurrence d'aujourd'hui. Or, cette présentation masque le fonctionnement réel des indexicaux de temps ${ }^{28}$. Il serait en effet préférable de dire qu'aujourd'hui et le 14 juillet 2019 ont le même référent, car la phrase (30) peut être considérée comme vraie même si la personne qui parle ignore la date du jour. Et il n'est pas non plus nécessaire que le destinataire le sache pour juger si cette phrase est vraie.

27 Pour dans $x$ temps demain, le délai est de $x$ temps +1 jour, et, pour dans $x$ temps le $n$, il est de $x$ temps + le laps de temps qui sépare le jour de l'énonciation du jour $n$ du mois en cours.

28 Cf. Vuillaume (2018: 227-228). 
On a donc intérêt à considérer les indexicaux temporels et les dates calendaires comme deux systèmes de datation, l'un centré sur le moment de la parole, repère qui change donc d'un emploi à un autre, l'autre fondé sur un repère fixe.

Il s'ensuit que, pour vérifier l'exactitude de :

(31) Pierre était encore là il y a cinq minutes.

il est inutile d'associer il y a cinq minutes à une date calendaire suivie d'une indication horaire. Il suffit qu'on soit en mesure de connaitre le laps de temps qui s'est effectivement écoulé entre le moment où Pierre était encore présent et le moment où est énoncée l'expression il y a cinq minutes, et il en irait de même, mutatis mutandis, pour dans x temps.

Cette propriété n'est pas altérée par le passage au style indirect. Dans l'exemple cidessus (28), le destinataire des paroles de la marquise n'a pas besoin de savoir à quelle heure M. de Norpois s'est engagé à revenir au bout de vingt minutes. La référence de dans $x$ temps est calculée à partir de $\mathrm{t}_{\mathrm{o}}$ (le moment de l'emploi de dans $x$ temps), et rien n'impose d'assigner à $t_{o}$ une place dans le système de datation calendaire. Quant au degré de précision avec lequel peut être spécifié le délai défini par $x$ temps, il n'est soumis théoriquement à aucune limitation, mais il est pratiquement limité au temps supposé requis pour articuler dans $x$ temps $^{29}$.

Dans $x$ temps se distingue donc de $x$ temps plus tard par trois traits (le second et le troisième découlant du premier) : i) la nature énonciative du repère auquel il renvoie ii) l'autonomie du système de repérage dont il relève iii) la précision de la datation qu'il autorise.

\section{Conclusion ou quand la langue fait du bricolage}

Tout compte fait, dans $x$ temps se distingue des authentiques indexicaux principalement par son comportement dans le discours indirect dépendant. Cette différence s'explique par les contraintes auxquelles est soumis $x$ temps plus tard, qui ne peut s'employer qu'aux deux conditions suivantes : i) la présence dans le contexte discursif amont d'un repère temporel récupérable par anaphore, et ii) connu avec un degré de précision au moins égal à celui de l'unité de temps utilisée pour définir le laps de temps dénoté par $x$ temps. Ainsi, dans l'exemple (28), c'est l'absence de repère contextuel qui fait obstacle à l'emploi de vingt minutes plus tard, alors que dans :

(32) Un jour, Pierre annonça de but en blanc à sa famille qu'il s'en irait *deux heures plus tard/le lendemain/trois jours plus tard/un mois plus tard... et ne reviendrait jamais.

la très vague indication fournie par un jour suffit à légitimer l'emploi de toutes les expressions anaphoriques faisant appel à une unité de temps au moins égale au jour, mais n'autorise pas le recours à deux heures plus tard. Il suffirait du reste, pour que l'emploi de cette expression soit possible, que le moment de la déclaration de Pierre soit plus précisément situé :

(32a) Un jour, au moment où il prenait son petit déjeuner, Pierre annonça de but en blanc à sa famille qu'il s'en irait deux heures plus tard et ne reviendrait jamais.

$\mathrm{X}$ temps plus tard est en outre inapte à transposer dans $x$ temps lorsqu'il $\mathrm{y}$ a, au sein même du fragment de discours rapporté, un candidat au rôle d'antécédent. Soit à mettre au discours indirect la phrase suivante :

(33) Pierre partira dès que possible, et Jean se mettra en route dans deux heures.

La transposition de dans deux heures ne peut manifestement se faire qu'au moyen de ... dans deux heures, car la référence de deux heures plus tard serait automatiquement calculée par

$29 C f$. Vuillaume $(1990: 19-20)$ et, sur les contraintes propres à la communication écrite, Vuillaume (2008 : 436-438). 
rapport au départ de Pierre, alors que dans deux heures dans (34) est évalué par rapport au moment de son énonciation :

(34) Au terme d'une longue discussion, il fut convenu que Pierre partirait dès que possible et que Jean se mettrait en route dans deux heures ( $\neq$ deux heures plus tard).

Pour que notre hypothèse soit crédible, il faudrait cependant expliquer aussi pourquoi demain - en dépit des contraintes qui concernent le lendemain et qui sont analogues à celles qui s'appliquent à $x$ temps plus tard - ne se comporte pas comme dans $x$ temps dans le discours indirect. Mais, sur ce point, nous ne pouvons formuler que des conjectures.

Le discours indirect décrit ce qu'on pourrait appeler des événements énonciatifs, qui ont la particularité d'occuper un laps de temps généralement assez court et, en tout état de cause, inférieur à un jour. Or, dans un récit, la plupart des événements peuvent être rattachés à un jour donné (que celui-ci soit, ou non, précisément situé dans le calendrier des événements narrés), et cela suffit à permettre l'emploi d'un anaphorique reposant sur une unité de temps égale ou supérieure au jour :

(35) Bientôt, une seule question demeura entre Nana et le comte : l'argent. Un jour, après lui avoir promis formellement dix mille francs, il avait osé se présenter les mains vides, à l'heure convenue. $[\ldots]$ Un tel manque de parole, tant de gentillesses perdues la jetèrent dans une rage de grossièretés. Elle était toute blanche.

- Hein ? tu n'as pas la monnaie... Alors, mon petit mufe, retourne d'où tu viens, et plus vite que ça! En voilà un chameau ! Il voulait m'embrasser encore !... Plus d'argent, plus rien ! tu entends ! Il donnait des explications, il aurait la somme le surlendemain. Mais elle l'interrompit violemment. (Zola, Émile, Nana)

Il s'agit certes d'une donnée contingente, mais dont il ne faudrait pas pour autant sous-estimer l'importance.

Il y a aussi, entre demain et dans $x$ temps, une autre différence qui, elle, relève de la diachronie : c'est que l'appartenance de demain à la classe des indexicaux est beaucoup plus ancienne que l'émergence du sens actuel de dans $x$ temps. Il se pourrait donc que dans $x$ temps, qui à date ancienne, n'avait aucune affinité particulière avec l'indexicalité, ait évolué en fonction des besoins de la langue. Cette hypothèse, dont la vérification exigerait une enquête minutieuse, paraît cependant plausible dans la mesure où le trait $<+$ indexical $>$ peut être une caractéristique occasionnelle de certains signes, comme en témoigne plus tard, qui se comporte, tantôt comme un indexical :

(36) À plus tard!

tantôt comme un anaphorique :

(37) Cette maison a été construite au XIXe siècle, mais le dernier étage a été ajouté plus tard.

Mais nous ne nous sommes déjà que trop aventurés dans le domaine des spéculations et nous laissons aux spécialistes de diachronie le soin de confirmer ou d'infirmer notre hypothèse.

\section{Bibliographie}

Apothéloz, Denis (2010). «L'opacité référentielle : Paramètres et statuts discursifs », in Colas-Blaise, M., Kara, M., Perrin, L., Petitjean, A. (éds.), La question polyphonique (ou dialogique) en sciences $d u$ langage (Recherches linguistiques $\mathrm{n}^{\circ} 31$ ), Metz : CELTED, 135-155.

Berthonneau, Anne-Marie (1989). Composantes linguistiques de la référence temporelle : les compléments de temps, du lexique à l'énoncé, Thèse de Doctorat d'État, Paris VII.

Blin, Georges (1954). Stendhal et les problèmes du roman, Paris : Librairie José Corti.

Bourgeois-Gironde, Sacha (2000). McTaggart: temps, éternité, immortalité, suivi de Trois essais de John McTaggart, Paris : Éditions de l'Éclat. 
Cornulier, Benoît de (1984). «Réversibilité : effets de rétroaction sémantique », L'Information Grammaticale, $\mathrm{n}^{\circ}$ 22, 3-6.

Douay Françoise, Pinto Jean-Jacques (1991). « Analogie/anomalie. Reflet de nos querelles dans un miroir antique », Communications, 53.

Gardies, Jean-Louis (1975). La logique du temps, Paris : Presses Universitaires de France.

Genette, Gérard (1972). Figures III, Paris : Éditions du Seuil.

Henry, Albert (1968). C'était IL Y A des lunes. Étude de syntaxe française, Paris : Klincksieck.

Kiparsky, Paul \& Kiparsky, Carol (1971). «Fact», in Steinberg, Danny D. \& Jakobovits, Leon A. (eds), Semantics. An Interdisciplinary Reader in Philosophy, Linguistics and Psychology, Cambridge : Cornell University Press

Kleiber, Georges (1986). « Déictiques, embrayeurs, "token-reflexives", symboles indexicaux, etc. : comment les définir? », L'information grammaticale, $\mathrm{n}^{\circ} 30$.

Klum, Arne (1961). Verbe et adverbe. Étude sur le système verbal indicatif et sur le système de certains adverbes de temps à la lumière des relations verbo-adverbiales dans la prose du français contemporain, Stockholm - Göteborg - Uppsala : Almqvist \& Wiksell.

Moeschler, Jacques (1993). «Aspects pragmatiques de la référence temporelle : indétermination, ordre temporel et inférence », Langages, $\mathrm{n}^{\circ} 112,39-54$.

Nilsson-Ehle, Hans (1943/44). «Le conditionnel 'futur du passé' et la périphrase devait + infinitif », Studia Neophilolologica 16, 50-58.

Nisard, M. (éd.) (1845). Macrobe, Varron et Pomponius Méla, Paris : J.J. Dubouchet et Compagnie, Éditeurs (Collection des auteurs latins avec la traduction en français).

Pier, John \& Schaeffer, Jean-Marie (éds.) (2005). Métalepses. Entorses au pacte de la représentation, Paris : Éditions de l'École des Hautes Études en Sciences Sociales.

Pinchon, Jacqueline (1974). «"L'homme dans la langue", l'expression du temps », Langue française, $\mathrm{n}^{\circ} 21$.

Recanati, François (1981). Les énoncés performatifs. Contribution à la pragmatique, Paris : Éditions de Minuit, 175-181.

Reichenbach, Hans (1966 (1947)). Elements of Symbolic Logic, The First Free Press Paperback Edition 1966, New York • London : The Free Press.

Rescher, Nicholas \& Urquhart, Alasdair (1971). Temporal Logic, Wien New York : SpringerVerlag.

Schlenker, Philippe (2005). «Sens et contexte », in Bourgeois-Gironde, Sacha (éd.), Les formes de l'indexicalité. Langage et pensée en contexte, Paris : Éditions de la rue d'Ulm.

Searle, John R. (1982). Sens et expression. Études de théories des actes de langage, Paris: Éditions de Minuit, 41-42.

Vuillaume, Marcel (1990). Grammaire temporelle des récits, Paris : Éditions de Minuit.

Vuillaume, Marcel (1993). «Le repérage temporel dans les textes narratifs », Langages, $\mathrm{n}^{\circ} 112,92-105$.

Vuillaume, Marcel (2000). "La signalisation du style indirect libre », in Mellet, Sylvie \& Vuillaume, Marcel (éds), Le style indirect libre et ses contextes, Amsterdam - Atlanta, GA : Rodopi, 107-130.

Vuillaume, Marcel (2008). «La temporalité discursive », in Birkelund Merete, Mosegaard Hansen Maj-Britt, Norén Coco (éds), L'énonciation dans tous ses états. Mélanges offerts à Henning Nølke à l'occasion de ses soixante ans, Bern • Berlin • Bruxelles... : Peter Lang, 433-450.

Vuillaume, Marcel (2018). «Deixis, anaphores, niveaux de fiction et style indirect libre », in Saussure, Louis de (éd.), Deixis et anaphore, London : iste editions, 225-242. 